\title{
Head Injury with Concurrent Cervical Spine Injury
}

\author{
Julius Kiboi, ${ }^{1}$ Ahmednasir Omar, ${ }^{1}$ Mohamed Ali Omar ${ }^{2}$ \\ ${ }^{1}$ School of Medicine University of Nairobi \\ ${ }^{2}$ Mbagathi District Hospital, Nairobi, Kenya \\ Correspondence to: Dr. Julius Kiboi, PO Box 51612-00200, Nairobi; email:j_kiboi@yahoo.com
}

\begin{abstract}
Dual diagnoses of moderate or severe head injuries occurring concurrently with cervical spine injuries are postulated to have very poor outcomes. These outcomes are unknown in resource-limited settings. Methods: Patient files with dual diagnoses between 2012 and 2016 at the Kenyatta National Hospital (KNH) were reviewed retrospectively. Clinical and outcome parameters were identified for each case and SPSS version 20 was used for correlation and regression tests. Results: Eleven patient files were identified; patients' median age was 34 years. $\mathrm{C} 2$ was the modal cervical spine injury level. The most common clinical presentation (63\%) was loss of consciousness; $45 \%$ of patients had a Glasgow Outcome score $\leq 1$. Using Pearson correlation study, age was significantly positively correlated with outcome: $r=$ $0.751, n=11, \mathrm{p}=0.008$. A significant regression equation was found $(F(1,9)=11.624, p<0.001)$ with an adjusted $R 2$
\end{abstract}

\section{Introduction}

The incidence of traumatic brain injury (TBI), the leading cause of death in all age groups, is postulated to be in the range of $20-30 \%$ (1). Estimates show TBI affects over 10 million people annually, leading to either hospitalization or mortality (2). The burden is worse in low and middle income countries (LMIC) because of the preponderance of risk factors associated with TBI and of health systems inadequately prepared to address the outcomes.

Sub-Saharan Africa demonstrates a higher TBI-related incidence rate of 170/100,000 owing to road traffic injuries relative to the global rate of $106 / 100,000$ (2). The rate of road traffic accidents in Kenya is 68 per 100,000 population, and traumatic brain injuries account for $50 \%$ of this figure (3). Other factors that increase the rate of road traffic injuries in this setting include flouting of traffic rules by road users, inadequate protective gear such as helmets or lack of their use by road users, and delayed response times by emergency medical personnel because of shortages or failures within the system.

Over the last 50 years, the average annual incidence of concomitant TBI and spinal cord injuries has increased (4). Studies show that in trauma patients with known head injury, of 0.515 to predict outcome on the Glasgow Outcome Scale based on age. Conclusion: For patients with dual diagnoses in a resource-limited setting, survival rate is $45 \%$; and older patients have poorer outcomes. Neurosurgeons practising in areas with limited resources should keep this in mind with the hope of attaining better outcomes.

Key words: Head injury, Dual diagnosis, Cervical spine injury

Ann Afr Surg. 2019; 16(1):11-15

DOI: http://dx.doi.org/10.4314/aas.v16i1.3

\section{Conflicts of Interest: None}

\section{Funding: None}

(C) 2019 Author. This work is licensed under the Creative Commons Attribution 4.0 International License.

simultaneous cervical spine injury occurs in $4-8 \%$ of the cases $(5,6)$. Cervical spine injuries occurring concurrently with moderate and severe head injuries typically worsen the patient's condition and have poorer outcomes in resourcepoor settings in LMIC. The most frequently involved area in the cervical spine is between the occiput and the $\mathrm{C} 3$ region (7). Patients with upper cervical injury are at a greater risk of suffering from skull base fractures and severe intracranial hematomas than those with mid to lower cervical injury (8). Approximately one-third of patients with cervical spine and/ or cord injuries have moderate or severe head injuries $(6$, $8)$. Injuries to the cervical spine are critical and may result in poor outcomes because of the anatomical proximity to the centers for control of respiration and cardiovascular activity and the reticular activating system. Cervical injuries may also compound the effects of head injuries through loss of diaphragmatic innervation with subsequent inadequate respiratory effort or spinal cord injury, resulting in neurogenic shock from loss of sympathetic outflow (5). Thus, survival of patients is hampered.

Currently, there is limited local data on presentation and outcome of patients who have dual injuries in our local settings. This retrospective study focused on elucidating the 
demographic characteristics of patients with dual diagnoses of moderate or severe head injury occurring concurrently with cervical spine injury, the mechanisms of injury, management strategies, and overall outcomes. It is hoped that this study will form a basis of reference and further research on patients with dual diagnoses.

\section{Methods}

\section{Patient population}

Records of patients diagnosed with moderate or severe head injury occurring concurrently with cervical spine injury between 2012 and 2016 at the Kenyatta National Hospital (KNH) were reviewed retrospectively for clinical, radiographic, and outcome data.

The outcomes of patients were recorded immediately at the time of discharge from hospital or at death postintervention. Because of the retrospective limitations of the study, data recorded from the files did not allow the study of outcomes 3-6 months postdischarge as some patients were lost to follow-up while others died, and therefore the immediate outcomes were recorded.

Characteristics identified were: age, sex, level of education, admission GCS, mechanism of injury and the preceding event, level of cervical spine injury, treatment administered, GCS at the time of departure from hospital, and final outcome documented using the Glasgow Outcome Scale.

The study was approved by the Kenyatta National HospitalUniversity of Nairobi Ethical and Research CommitteeERC (Study approval number UP957/12/2016). The ERC waived the requirement for informed consent because of the retrospective nature of the study.

\section{Statistical analysis}

Data were analyzed using SPSS version 20 (IBM Corporation). Age, admission GCS, GCS prior to discharge or death, and overall outcome were evaluated as continuous variables. Patient characteristics were summarized using means, medians, range and frequencies for continuous variables; the mode was used to summarize categorical variables. The Pearson product-moment correlation identified correlations between variables. A simple linear regression analysis predicted outcomes using variables that had a significant correlation.

\section{Results}

Data from 2012 to 2016 were obtained from the Records Department at KNH. We retrieved 1,432 patient files that had a diagnosis of head injury. Of these cases, 358 patients were classified as having moderate or severe head injuries; dual presentation was diagnosed in only 11 patients or $0.03 \%$ of the patient group.

\section{Demographic data}

Patients' ages ranged from 5 to 60 years. The mean age was 27.2 years, median age 34 years, and modal age group between 0 and 20 years. Male patients constituted $81.8 \%$ of the total patient population. More than $72 \%$ of patients resided within Nairobi County and its environs. Only 2 patients had attained secondary school education.

\section{Clinical presentation}

The most common clinical presentation was loss of consciousness, present in $63 \%$ of the patient population. Aphasia and quadriplegia were noted in 9 and $27 \%$ of patients respectively. Other clinical presentations were gait disturbance, otorrhea, bleeding from the ear, quadriparesis, aphasia and incontinence of both urine and stool (Figure 1). The GCS of the patients ranged from 3 to 12. Modal GCS at presentation were 8 and 10 , recorded in 3 patients each. Mean GCS at presentation was 8.6 and median was 9 . Prior to presentation at the hospital, the preceding events were reported: speeding, lack of observing traffic rules, intoxication, and physical violence. The most common mechanism of injury was fall from height, accounting for $36 \%$ of injuries, next was motor cycle accidents $27 \%$, assault $18 \%$, passenger in an automobile accident $9 \%$, and

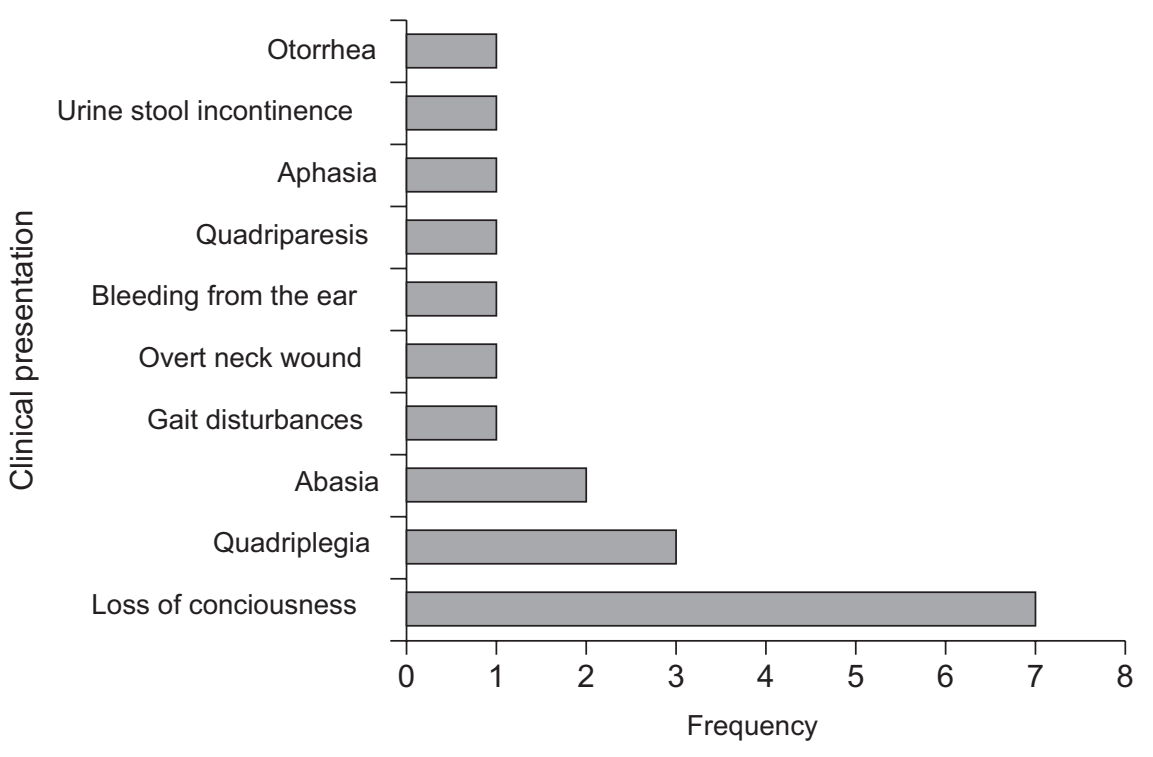

Figure 1: Frequency of various clinical presentations 
pedestrian versus motor vehicle $9 \%$.

The most common area of injury in the cervical region was at $\mathrm{C} 2$ level, seen in $45 \%$ of the patients; $18 \%$ of patients had injury at $\mathrm{C} 3$ level, $18 \%$ at $\mathrm{C} 4$ level, $9 \%$ at $\mathrm{C} 1$ level, and $9 \%$ at $\mathrm{C} 7$ level. Six patients had multi-level injuries in the cervical region, with some being continuous segmental injuries and others skip injuries or different forms of injury such as a fracture and loss of cervical spine alignment.

\section{Management}

Four patients $(36 \%)$ each were managed by skull traction and hard collar respectively, 2 patients (18\%) were managed using a soft collar, $1(9 \%)$ patient was symptomatically managed and observed before being discharged, 1 (9\%) patient was managed using a combination of skull traction and a soft collar. No patient underwent surgical intervention or was initiated on steroids.

\section{Period of hospitalization}

The period of hospitalization was categorized into: $0-2$ weeks, 2-4 weeks, 4-6 weeks (Table 2). The mean period of hospital stay before discharge or death was 2.5 weeks; the modal and median range was $0-2$ weeks.

\section{Discharge from hospital}

GCS at the time of leaving hospital was varied with mean GCS at 7.2 and modal and median GCS at 3 each. Five patients were discharged with GCS at 15; the rest were mortalities with GCS score of 3 each. Four patients had complete spinal recovery, 5 patients were quadriplegic and were all mortalities, and 1 patient had partial recovery with the ability to use walking aids. The overall outcome was documented using the Glasgow Outcome Scale with 55\% of the patients having a score of $5,36 \%$ a score of 1 , and $9 \%$ a score of 2 (Table 1). This outcome is the immediate post-discharge.

Table 1: Overall outcome of the patients

\begin{tabular}{lcc}
\hline $\begin{array}{l}\text { Score on the Glasgow Outcome } \\
\text { Scale }\end{array}$ & $\begin{array}{l}\text { No. of } \\
\text { patients }\end{array}$ & $\begin{array}{l}\% \\
\text { contribution }\end{array}$ \\
\hline - Asymptomatic & 0 & 0 \\
$\begin{array}{l}\text { - Symptomatic but completely } \\
\text { ambulatory }\end{array}$ & 4 & 36 \\
$\begin{array}{l}\text { - Symptomatic, }<50 \% \text { in bed } \\
\text { during the day }\end{array}$ & 1 & 9 \\
3- Symptomatic, $>50 \%$ in bed, & & \\
$\quad$ but not bedbound & 0 & 0 \\
4 - Bedbound & 0 & 0 \\
5 - Death & 6 & 55 \\
Total & 11 & 100 \\
\hline
\end{tabular}

Pearson product-moment correlation coefficient assessed relationships between admission GCS and discharge GCS, admission GCS and outcome, level of cervical spinal injury and outcome, and age and outcome, and identified those that are statistically significant at $\mathrm{p}=0.05$. A positive correlation was identified between age and outcome $(r=0.751, n=11$, $\mathrm{p}=0.008)$. Preliminary results show a partial Pearson correlation while controlling for admission GCS ( $r=0.644$, $n=11, \mathrm{p}=0.044)$ and level of cervical spine injury $(r=0.724$, $n=11, \mathrm{p}=0.018$ ). A positive correlation exists between age and outcome even while the effect of admission GCS and level of cervical spine injury was controlled.

No significant correlation was identified between the other variables and between the different diagnoses. A moderately strong positive correlation was seen between age and outcomes documented using the Glasgow Outcome score. Increases in age were correlated with increases in higher scores on the Glasgow Outcome Scale, thus portending worse outcomes.

A simple linear regression predicted outcome on the Glasgow Outcome Scale based on age of the patients. A significant regression equation was found $(\mathrm{F}(1,9)=11.624, \mathrm{p}<0.001)$ with an adjusted $\mathrm{R} 2$ of 0.515 . Patients' predicted age is equal to a score of $0.770+0.082$ (age) on the Glasgow Outcome Scale when age is measured in years. The outcome increased 0.082 for each year of age.

\section{Discussion}

Head injuries overall are $80 \%$ mild, $10 \%$ moderate and $10 \%$ severe (1). Studies show that the probability of concomitant head injury increases in settings of GCS scores lower than $8(5,9)$. The focus group of this study was patients who lie on the lower end of the spectrum in the GCS and have concurrent cervical spine injury. The overarching factor is the existing resource limitations in this setting in which these patients were managed that leads to a trifecta that portends poor outcomes.

Studies are also limited by the rare occurrence. In the 5-year period that was reviewed, only 11 patients fitted strictly into our inclusion criteria. This rarity could be because of the severity of the injuries occurring and death ensuing before presentation to an emergency setting, resulting from delays within the system.

It is also surmised that patients who make it to hospital in this resource-limited setting generally have very poor outcomes due to the severity of their injuries. Our findings challenge this assumption by demonstrating that $45 \%$ of the patients survived and had Glasgow Outcome scores $\leq$ 
1. To the best of our knowledge this study is the first of its kind within this region.

The demographics of the patient group showed that age ranged from 5 to 60 years, with males making up over $80 \%$ of the group. This figure concurs with worldwide studies that show males generally constitute a higher percentage of those exposed to trauma (10). Several mechanisms of injury have been implicated in simultaneous cervical injury and head injury patients. The commonest injuries are motor vehicle accidents, automobile versus pedestrian accidents, motorcycle accidents, falls, assaults and gunshot wounds, in that order (5). In our patient cohort, fall from height and motorcycle accidents were most common, and motor vehicle accidents and automobile versus pedestrian contributed $9 \%$ each as the mechanisms of injury leading to dual diagnoses. Traumatic intracranial hemorrhage is a common finding in moderate and severe head injuries; however, it is not a predictor of occult cervical spine injury (11). Surprisingly, none of our patients underwent surgery as internal bleeds or raised intracranial pressures were absent. All our patients underwent conservative management. In associated injuries, surgery of the head and immobilization of the spinal fracture are carried out as the first step; realignment of the spine with possible decompression and stabilization of the affected segment is a second step (12).

Cervical spine injuries can be treated using conservative methods in the initial management as an adjunct to surgical management or as definitive management. Skeletal skull traction is indicated mainly in cases of facet subluxation or dislocation and in burst-type fractures to stabilize and realign the cervical spine (13). Traction is contraindicated in distractive injuries or in patients with certain skull fractures. The timing of skull traction still remains controversial (13). Halo vests restrict up to $75 \%$ of flexion-extension at $\mathrm{C} 1-\mathrm{C} 2$ and offer superior control of lateral bending and rotation, and are therefore the orthosis of choice when rigid immobilization is required of an unstable spine injury.

The combined injuries present a dilemma: many of the drugs used to treat SCI may interfere with cognitive rehabilitation and, conversely, drugs that are used to control seizures in TBI patients may undermine locomotor recovery after SCI (14). A concomitant injury also increases the immune response several-fold with a more profound increase in the spinal cord relative to the brain and, therefore, contributing to the overall long-term morbidity and mortality (15).

Progesterone exhibits neuroprotective effects in TBI and SCI by activating autophagy in cortical astrocytes protecting against motor neuron degeneration and might have been helpful to our patient group (16).
The patients who died in our cohort could be those who had more severe injuries, such as diffuse axonal injuries, that could not be identified using the available resources. Diffusion tensor imaging-derived fractional anisotropy is a sensitive tool to detect TBI-related white matter damage in patients with dual diagnoses, and would have been useful (17).

The positive correlation between age and final outcome is significant: younger patients in this patient cohort had better outcomes than older patients. The significant positive correlation was still maintained even when the admission GCS and level of cervical spine injury were controlled for using partial correlation. Therefore, older aged patients had poorer outcomes in this setting when they had dual diagnoses. The incidence of spinal injuries in concomitant injury increases dramatically with age, with elderly patients 21 times more likely to suffer spinal trauma than pediatric populations (6).

Correlation between admission GCS and GCS prior to discharge or death was not significant. This correlation points towards difficulty in predicting final outcomes based on admission GCS, and underlines the importance of evaluating prognosis on day-to-day GCS scores and response to therapy. None of the other independent variables had significant correlations with outcome when evaluated. For patients who were discharged, concomitant injury led to increased rehabilitation costs, poor neuropsychological test performances and greater demands on clinician resources than injuries in a single spectrum (18). Recovery of function after cervical SCI is retarded by a moderate TBI in the contra lateral hemisphere (14). A high level of social support is vital to help these patients in their daily lives (19). In resource-limited settings, such as in Kenya, where support is inadequate, the risk is real in dual diagnoses patients of losing out on paid employment, concomitant emotional changes and increased dependence.

\section{Conclusion}

This study describes a patient cohort over a 5-year period with dual diagnoses of either moderate or severe head injury occurring concurrently with cervical spine injury, and the outcomes.

The limitations of a retrospective study exist particularly in the selection bias, possibility of missing information from records, low numbers for inclusion in the study, and loss to follow-up in resource-limited settings.

The diagnosis of moderate and severe head injury with concurrent cervical spine injury in patients in resourcelimited settings portends a grim outlook on outcomes. 
Despite the low numbers, our findings suggest a $45 \%$ chance of outcomes $\leq 1$ on the Glasgow Outcome Scale. Neurosurgeons practising in LMIC with resource limitations should keep this in mind with the hope of attaining better outcomes.

\section{Acknowledgements}

The authors acknowledge the University of Nairobi, the Department of Surgery and staff at the Records Department at Kenyatta National Hospital for facilitating easy and smooth collection of data for publishing this work.

\section{References}

1. Nazir M, Khan SA, Raja RA, et al. Cervical spinal injuries in moderate to severe head injuries. J Ayub Med Coll Abbottabad. 2012; 24:3-4.

2. Hyder AA, Wunderlich CA, Puvanachandra P, et al. The impact of traumatic brain injuries: A global perspective. Neuro Rehabilitation. 2007; 22(5):341-53.

3. Kinyanjui B. Traumatic brain injury in Kenya: A preliminary review of the literature. SAGE Open. 2016; 6(1):2158244016638392.

4. Hagen EM, Eide GE, Rekand T, et al. Traumatic spinal cord injury and concomitant brain injury: A cohort study. ActaNeurol Scand. 2010; 122:51-7.

5. Holly LT, Kelly DF, Counelis GJ, et al. Cervical spine trauma associated with moderate and severe head injury: Incidence, risk factors, and injury characteristics. J Neurosurg Spine. 2002; 96(3):285-91.

6. Paiva WS, Oliveira AM, Andrade AF, et al. Spinal cord injury and its association with blunt head trauma. Int J Gen Med. 2011; 4:613-5.

7. Tian H-L, Guo Y, Hu J, et al. Clinical characterization of comatose patients with cervical spine injury and traumatic brain injury. J Trauma Acute Care Surg. 2009; 67(6):130510.
8. Iida H, Tachibana S, Kitahara $\mathrm{T}$, et al. Association of head trauma with cervical spine injury, spinal cord injury, or both. J Trauma Acute Care Surg. 1999; 46(3):450-2.

9. Hills MW, Deane SA. Head injury and facial injury: Is there an increased risk of cervical spine injury? J Trauma. 1993; 34(4):549-53.

10. Santamariña-Rubio E, Pérez K, Olabarria M, et al. Gender differences in road traffic injury rate using time travelled as a measure of exposure. Accid Anal Prev. 2014; 65:1-7.

11. Frye G, Wolfe T, Knopp R, et al. Intracranial hemorrhage as a predictor of occult cervical spine fracture. Ann Emerg Med. 1994; 23(4):797-801.

12. Steudel WI, Rosenthal D, Lorenz R, et al. Prognosis and treatment of cervical spine injuries with associated head trauma. Acta Neurochir Suppl (Wien). 1988; 43:85-90.

13. Lauweryns P. Role of conservative treatment of cervical spine injuries. Eur Spine J. 2010; 19(1):23-6.

14. Inoue T, Lin A, Ma X, et al. Combined SCI and TBI: Recovery of forelimb function after unilateral cervical spinal cord injury (SCI) is retarded by contralateral traumatic brain injury (TBI), and ipsilateral TBI balances the effects of SCI on Paw placement. Exp Neurol. 2013; 248:136-47.

15. Anthony DC, Couch Y. The systemic response to CNS injury. Exp Neurol. 2014; 258:105-11.

16. Kim J, Kim T-Y, Cho K-S, et al. Autophagy activation and neuroprotection by progesterone in the G93A-SOD1 transgenic mouse model of amyotrophic lateral sclerosis. Neurobiol Dis. 2013; 59:80-5.

17. Wei CW, Tharmakulasingam J, Crawley A, et al. Use of diffusion-tensor imaging in traumatic spinal cord injury to identify concomitant traumatic brain injury. Arch Phys Med Rehabil. 2008; 89(12):S85-S91.

18. Bradbury CL, Wodchis WP, Mikulis DJ, et al. Traumatic brain injury in patients with traumatic spinal cord injury: Clinical and economic consequences. Arch Phys Med Rehabil. 2008; 89(12):S77-S84.

19. Boyle CL, Nott MT, Baguley IJ, et al. Contextual influences on employment of people with dual diagnosis: Spinal cord injury and traumatic brain injury. Aust Occup Ther J. 2014; 61(5):335-43. 